\title{
Kernos
}

Revue internationale et pluridisciplinaire de religion grecque antique

7| 1994

Varia

\section{Nouveau regard sur les Grandes Déesses de Mégalopolis : influences, emprunts, syncrétismes religieux}

\section{Madeleine Jost}

\section{(2) OpenEdition}

\section{Journals}

\section{Édition électronique}

URL : http://journals.openedition.org/kernos/1101

DOI : 10.4000/kernos. 1101

ISSN : 2034-7871

\section{Éditeur}

Centre international d'étude de la religion grecque antique

\section{Édition imprimée}

Date de publication : 1 janvier 1994

ISSN : 0776-3824

\section{Référence électronique}

Madeleine Jost, « Nouveau regard sur les Grandes Déesses de Mégalopolis : influences, emprunts, syncrétismes religieux », Kernos [En ligne], 7| 1994, mis en ligne le 20 avril 2011, consulté le 01 mai 2019. URL : http://journals.openedition.org/kernos/1101; DOI : 10.4000/kernos.1101 
Kernos, 7 (1994), p. 119-129.

\section{NOUVEAU REGARD SUR LES GRANDES DÉESSES DE \\ MÉGALOPOLIS : INFLUENCES, EMPRUNTS, SYNCRÉTISMES RELIGIEUX}

Par Grandes Déesses j'entends non pas les divers couples de déesses au sens large, mais la réalité religieuse spécifique de Mégalopolis, où Mégalai Théai est une appellation cultuelle liée à un sanctuaire des Grandes Déesses, Déméter et Koré selon Pausanias' ${ }^{1}$. Ce sera l'objet de cette communication.

La fondation de Mégalopolis, créée ex nihilo en 370/369 av. J.-C., s'accompagna d'une véritable politique cultuelle. L'objectif en était de mettre en place des divinités qui soient aptes à éveiller chez les nouveaux citoyens, déplacés parfois contre leur gré de leur bourgade d'origine, la conscience d'appartenir à une communauté. Les nouveaux cultes reflètent-ils des influences? (On a invoqué les cultes de Thèbes, en raison du rôle des Thébains dans la création de la nouvelle cité). Ont-ils emprunté aux cultes de la chôra de Mégalopolis, ou à des sanctuaires extérieurs particulièrement populaires comme Éleusis? Ont-ils créé des divinités plus ou moins syncrétiques? Entre syncrétisme réel et simple "influence» superficielle, ou "emprunt", on peut quelquefois hésiter. Je voudrais donc, à propos des cultes de l'enclos des Grandes Déesses de Mégalopolis, étudier la mise en œuvre de ces différentes modalités de contact lors de la création du sanctuaire. Le péribole des Grandes Déesses se trouvait dans le secteur Sud-Ouest de l'agora; il a été ruiné par un affluent de l'Hélisson et n'est connu que par la description de Pausanias ${ }^{2}$. Il renfermait, à côté des Grandes Déesses, une quantité de divinités : ce sont une partie de ces cultes qui fourniront la base de notre réflexion.

1 Paus., VIII, 31, 1. Sur les Grandes Déesses, voir R. STIGLITZ, Die Grossen

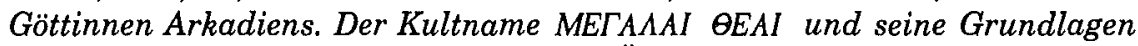
(Sonderschriften herausgegeben vom Österreichischen Archäologischen Institut in Wien, Band XV, 1967) et, à propos de cet ouvrage, M. JosT, Les Grandes Déesses d'Arcadie, in REA, 77 (1970), p. 138-151; EAD., Sanctuaires et cultes d'Arcadie, Paris, 1985, p. 227-229 et 340-345.

2

Paus., VIII, 31, 1 - $31,8$. 
L'entourage immédiat des Grandes Déesses soulève une première série de questions. "Il y a, à côté de Déméter, écrit Pausanias, un Héraklès d'une coudée environ. Dans ses vers, Onomacrite range cet Héraklès parmi les êtres appelés Dactyles de l'Ida” ${ }^{3}$. La plupart des exégètes modernes admettent l'identification par Pausanias du petit Héraklès comme un Dactyle de l'Ida ${ }^{4}$ et ils considèrent que sa présence auprès des Grandes Déesses, et plus particulièrement à côté de Déméter, n'était pas fortuite 5 . On aimerait être sûr de l'identification de l'Héraklès en question, qui semble dictée à Pausanias par la petite taille de la statue et le rapprochement qu'il fait d'un passage d'Onomacrite. On aimerait aussi avoir plus de certitudes sur le caractère signifiant de son voisinage avec Déméter; il est vrai que l'agencement du texte de la Périégèse invite effectivement à considérer ensemble Déméter et Koré, Héraklès Dactyle, les deux canéphores qui précédaient le groupe cultuel et une table à offrandes, avant de passer au sanctuaire de Zeus Philios. Admettons comme une hypothèse vraisemblable que l'on est en droit de chercher une signification au groupement des Grandes Déesses avec Héraklès. Il reste à l'interpréter.

Certains ont voulu raisonner en terme d'«influence». B. Hemberg, dans son ouvrage sur les Kabires ${ }^{6}$, considère qu'Héraclès Dactyle est un des Grands Dieux, dont le nom apparaît à Mégalopolis dans une inscription du Ier s. av. J.-C. ${ }^{7}$ R. Stiglitz ${ }^{8}$, qui fait la critique de l'affirmation de B. Hemberg, pense que l'inclusion d'Héraklès Dactyle au monde cabirique, si elle a quelque réalité, ne peut être que récente et se surajouter à un couple antérieur. Il propose d'y voir l'«influence» du Kabirion de Thèbes où Déméter Kabeireia voisinait avec les Kabires ${ }^{9}$ : le couple Déméter - Héraklès ferait écho à la présence d'une Déméter Kabeireia tout près du Kabirion de Thèbes et de ses Grands Dieux. À la vérité, la notion d'influence est employée ici mal à propos: non seulement rien ne permet de savoir si Héraklès faisait partie des Grands Dieux, mais, plus encore, l'influence du Kabirion de Thèbes est

\footnotetext{
3 PAUS., VIII, 31, 3.

4 Cf. B. HEMBERG, Die Idaiischen Daktylen, in Eranos, 50 (1952), p. 41- 59.

5 R. STIGLITZ, op. cit. (n. 1), p. 25-26.

6 B. Hemberg, Die Kabiren, Uppsala, 1950, p. 46.

$7 \quad I G \mathrm{~V} 2,466$.

8 R. STIGLiTZ, op. cit. (n. 1), p. 26; cf. aussi p. 16 et 147.

9 PAUS., IX, 25, 5.
} 
peu vraisemblable; si Épaminondas a influencé la politique arcadienne, il en va bien autrement des phénomènes religieux qui impliquent de véritables contacts de population.

L'hypothèse d'une "influence" thébaine étant impossible à prouver, mieux vaut chercher en Arcadie même l'explication de cette association de Déméter et d'Héraklès Dactyle. Elle pourrait reproduire le schéma d'une vieille association cultuelle, rappelant l'association de Déméter et Héraklès Dactyle à Mykalessos en Béotie ou leur voisinage à Élis ${ }^{10}$ :

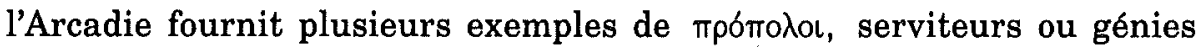
parfois armés, aux côtés d'une déesse. Ainsi, le géant Hoplodamos et ses compagnons avaient, selon la tradition de Méthydrion, assuré la protection de Rhéa contre Kronos à l'époque où elle se trouvait enceinte de Zeus ${ }^{11}$; Anytos, un Titan, père nourricier de Despoina ${ }^{12}$, pouvait avoir une fonction similaire à Lykosoura auprès de Déméter et Despoina, et les Kourètes et Korybantes ${ }^{13}$, qui apparaissaient dans le décor sculpté du trône des déesses et étaient associés à leur mystères, appartiennent à la même famille de трómo入or armés auprès d'antiques déesses; enfin les Géants d'Arcadie, qui avaient donné son nom de $\Gamma$ iravtls à la contrée ${ }^{14}$, avaient leur légende attachée à la région de Bathos, où ils auraient livré la gigantomachie près du sanctuaire des Grandes Déesses ${ }^{15}$. Si l'on admet qu'Héraklès Dactyle était non pas un Grand Dieu, mais un vieux génie de la nature ${ }^{16}$, on pourrait considérer sa présence dans le péribole des Mégalai Théai comme le prolongement de l'un de ces démons qui entouraient anciennement Déméter ou des déesses analogues, en Arcadie comme dans le monde préhellénique.

10 Paus., IX, 19, 5 et VI, 23, 3.

11 PAUS., VIII, 36, 2.

12 Paus. VIII, 37, 5-6. Cf. déjà F. VIAN, La guerre des géants. Le mythe avant l'époque hellénistique, Paris, 1952, p. 240.

13 PAus. VIII, 37, 5. À noter la présence d'un autel de la Mère à Lykosoura et le motif de lions et de tambourins qui ornait le tabouret des déesses (cf. PAUS, VIII, 37, 2 et les vestiges du groupe sculpté : E. LÉvY et J. MARCADÉ, in $B C H, 96$ [1972], p. 967-1004).

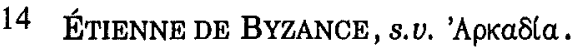

15 PAUs., VIII, 29, 1.

16 Cf. l'article de B. HEMBERG cité à la note 4 et C. BonNET, dans Melqart. Cultes et mythes de l'Héraclès tyrien en Méditerranée, Namur, 1988, p. 387. 
Au total, la présence d'Héraklès ne serait pas due à une «influence» thébaine, elle se situerait dans une catégorie religieuse dont on a d'autres exemples en Mégalopolitide. En l'occurrence, la notion d'influence est inadéquate : d'abord parce que supposer une influence religieuse à partir de données politiques est hasardeux; ensuite parce que parler en termes d'influence occulte ce qui fait l'originalité du phénomène religieux arcadien et la spécificité de ses cultes.

Le terme d'«emprunt» semble a priori plus facile à utiliser. Dans le péribole des Grandes Déesses sont implantées des divinités du panthéon grec, individualisées par une épiclèse locale : ainsi Aphrodite Machanitis qui a "suscité mille inventions et toutes sortes d'artifices de langage»17. Certaines peuvent avoir fait l'objet d'un culte dans l'une des bourgades qui précédèrent Mégalopolis; il s'agit en tout cas incontestablement «d'emprunts». Un cas particulier est représenté par les antiques xoana figurant Héra, Apollon et les Muses qui se trouvaient devant l'entrée du sanctuaire d'Aphrodite dans le péribole des Grandes Déesses : ils ont été, nous dit Pausanias, apportés de Trapézonte ${ }^{18}$. Ce transfert matériel, qui dépouille la ville d'origine, est un fait exceptionnel; Mégalopolis a le plus souvent établi dans la cité des «doublets" des grands sanctuaires de la chôra et de ses bourgades en respectant l'intégrité du sanctuaire originel : exemple la "réplique» du sanctuaire du mont Lycée sur l'agora ${ }^{19}$ ou la statue d' Hermès Akakésios (l'original continuait à se dresser dans la bourgade désertée d'Akakésion, tandis qu'un double en avait été exécuté pour Mégalopolis ${ }^{20}$ ). Mais les gens de Mégalopolis, exaspérés par le refus des gens de Trapézonte de quitter leur ville, massacrèrent un bon nombre d'entre eux ${ }^{21}$ et ils exercèrent un véritable pillage de Trapézonte. L'ancienneté des xoana (cf.

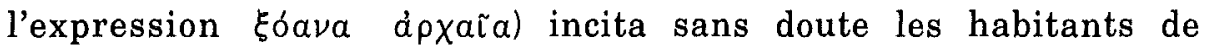
Mégalopolis à leur réserver une place de choix dans le péribole des Déesses. L'emprunt a donc ici un caractère rare : il dépouille le lieu

17 PAUS., VIII, 31, 6.

18 PAUS., VIII, 31, 5-6.

19 PAUS., VIII, 30, 2.

20 PAUS., VIII, 36, 10 et 30,5 . Pour cette politique des «doublets», voir M. JOST, Sanctuaires ruraux et sanctuaires urbains en Arcadie, in O. REVERDIN et B. Grange (éds), Sanctuaires, Fondation Hardt, Vandœuvres-Genève, Fondation Hardt, 1992 (Entretiens sur l'Antiquité classique, 37), p. 228-232.

21 PAUS., VIII, 27, 6. Les autres seraient partis vers le Pont. 
d'origine à la fois des objets du culte et du culte lui-même, il revêt une forme agressive.

Des cas plus complexes sont offerts par Zeus Philios et par les Grandes Déesses elles-mêmes. Pour Zeus Philios, le mot de «syncrétisme» vient immédiatement à l'esprit: «À l'intérieur du péribole se trouve un temple de Zeus Philios. Polyclète d'Argos est l'auteur de sa statue qui a l'allure d'un Dionysos : chaussé de cothurnes, il tient d'une main un vase a boire et de l'autre un thyrse sur lequel est posé un aigle ${ }^{22}$. Ce culte doit être une création du IVe siècle liée à la construction de la capitale : son développement en Grèce se situe dans cette période ${ }^{23}$. Le Polyclète en question serait donc ici Polyclète le Jeune dont on situe la carrière entre 400 et $360^{24}$. Zeus Philios est le dieu de l'amitié, dont on attendait l'heureuse influence sur les rapports entre citoyens. La représentation qu'avait donnée Polyclète du dieu est «syncrétique» au sens où elle "mélange» les attributs de deux divinités Dionysos et Zeus. Les cothurnes, la coupe et le thyrse évoquent Dionysos et rappellent que Zeus Philios devait être plus particulièrement, comme le suppose M. P. Nilsson ${ }^{25}$, le dieu du symposium, de l'amitié joyeuse et désordonnée des banquets où le vin de Dionysos coule à flot. Mais l'aigle de Zeus, attribut du dieu dans toute sa majesté, évoque en même temps l'idée d'une amitié plus grave, celle qui doit unir tous les hommes rassemblés dans la cité nouvelle: Zeus est le protecteur de l'ordre social. Y eut-il syncrétisme au sens propre, avec ce que cela implique de réinterprétation, de modification de la substance des deux modèles ${ }^{26}$ ? On peut le penser : le Zeus Philios de Mégalopolis était bien une divinité nouvelle et originale tenant sa convivialité avenante de Dionysos, et de Zeus la hauteur de ses aspirations.

Plus problématiques sont les Grandes Déesses à qui appartenait l'ensemble du sanctuaire. Pausanias précise à leur sujet: «Ce sont Déméter et Koré, comme je l'ai déjà indiqué dans le livre sur la

22 PAUS., VIII, $31,4$.

23 M. P. NILsson, Geschichte der griechischen Religion, $\mathrm{I}^{2}$, Munich, 1955, p. 808810.

Cf. D. ARnold, Die Polykletnachfolge, Berlin, 1969, p. 9.

M. P. Nilsson, loc. cit. (n. 23).

26 Cf. la mise au point de la notion de syncrétisme dans Éd. W ILL et Cl. OrRIEUX, Ioudaïsmos- Hellènismos, Nancy, 1986, p. 148. 
Messénie [ce qui est malheureusement inexact]. Koré est appelée Sôteira par les Arcadiens» 27. Ont-elles été «empruntées» à un site arcadien fondateur? Les Grandes Déesses sont attestées par Pausanias à «l'endroit nommé Bathos» 28 , près de Trapézonte dans la plaine de Mégalopolis, sans que l'on puisse savoir avec certitude si le sanctuaire a précédé celui de Mégalopolis ou s'il en dérive ${ }^{29}$ Sont-elles un "mélange», un syncrétisme, au sens courant du terme, de Déméter et d'une déesse indigène, comme le proposait $R$. Stiglitz dans son ouvrage sur les Mégalai Théai ${ }^{30}$ ? À moins encore qu'elles ne soient un simple «emprunt» à Éleusis, comme le suggère, on le verra, Pausanias.

L'origine de l'appellation de Grandes Déesses n'est pas possible à déterminer de manière sûre. Bathos mis à part, elle est attestée par Pausanias en Messénie à propos d'un culte que la loi religieuse d'Andania au Ier s. av. J.-C. désigne seulement comme celui de "Déméter et Hagna" 31 , ce qui rend peu vraisemblable l'idée d'un apport messénien en Arcadie. Si elle est mégalopolitaine, ce vers quoi penche R. Stiglitz ${ }^{32}$ sans preuves irréfutables, l'appellation de Mégalai Théai pourrait être inspirée de celle des Mégaloi Théoi de Mégalopolis et de la Mégalè Méter de Lykosoura ${ }^{33}$ et avoir été choisie en harmonie avec le nom de la Mégalè Polis. Au total, reconnaissons qu'il n'y a rien à tirer de l'appellation des Mégalai Théai pour connaître leur nature.

S'agit-il, comme le veut R. Stiglitz, d'un nouveau couple syncrétique, auquel se serait surajoutée l'image mère-fille, d'où l'explication par Déméter et Koré ? Les indices en ce sens sont pauvres : c'est essentiellement l'appellation Sôteira, précisée par Pausanias pour la seconde déesse, qui indiquerait une tonalité proprement arcadienne. L'appellation apparaît peut-être dans une dédicace du Ier s. ap. J.-C. trouvée à Lykosoura "en faveur de Despoina et Sôtira»; R. Stiglitz pense

27 PAUS., VIII, 31, 1.

28 Paus., VIII, 29, 1.

29 R. STIGLiTZ, op. cit. (n. 1), p. 17-30 et 50-54 évoque les deux hypothèses.

30 Ibid., p. 17-30 et 146-156.

31 PAUS., IV, 3, 10 et IG V 1, 1390.

32 Cf. note 29 .

33 Cf. note 7 pour les Grands Dieux (ils ne sont pas cités par Pausanias) et PAUS., VIII, 37, 3 pour la Grande Mère. 
que cette dernière est la déesse de Mégalopolis ${ }^{34}$. En fait, l'appellation de Sôteira, qui comporte surtout l'idée de sauvegarde de la communauté, se trouve employée pour Koré à Cyzique et à Sparte ${ }^{35}$; elle ne suffit donc pas à faire de Koré-Sôteira une divinité au caractère indigène nettement affirmé par rapport à Koré. À tout prendre, le cas des Grandes Déesses d'Andania, où la seconde déesse s'appelle Hagna, autoriserait plus de conclusions; mais l'appellation de Mégalai Théai y paraît bien tardive ${ }^{36}$. Quant aux Grandes Déesses de Bathos, la présence d'une source sur le site n'autorise pas à parler d'une ancienne nymphe pour la deuxième déesse, comme l'envisage $R$. Stiglitz ${ }^{37}$. L'originalité de la déesse-fille n'apparaît guère à travers son appellation locale.

Sôteira était-elle, comme Despoina à Lykosoura, plus importante que Déméter dans le couple des deux déesses, ce qui la démarquerait de la Koré éleusinienne ? La prééminence de Koré dans le péribole se marque par l'existence, outre le culte commun, d'un sanctuaire où elle est honorée seule : "La statue est en marbre, note Pausanias, haute de huit pieds; des bandelettes recouvrent entièrement le piédestal. Les femmes peuvent pénétrer dans ce sanctuaire en tout temps; les hommes n'y entrent qu'une fois l'an»38. Ceci rappelle le rôle dévolu à Koré lors des Koragia à Mantinée ou la prédominance de Despoina à Lykosoura ${ }^{39}$; mais ce n'est pas la preuve qu'elle ait tenu le premier rôle dans le couple des Grandes Déesses (à Mantinée, Koré seule voisine précisément avec un couple Déméter-Koré d'allure éleusinienne). Il n'y a rien à tirer non plus, pour connaître Sôteira, de la différence de technique entre les deux statues de culte, l'une en pierre, l'autre plus petite, acrolithe : il n'y a, comme je l'ai noté ailleurs ${ }^{40}$, aucune raison de penser avec $R$. Stiglitz que la seconde soit une ancienne statue de Despoina apportée à Mégalopolis.

34 IG V 2, 524; cf. R. STIGLitz, op. cit. (n. 1), p. 34 n. 72 (réserves exprimées par M. Jost in REA, 72 [1970], p. 147).

35 Pour Sparte, Paus., III, 13, 2; pour Cyzique, cf. F. W. Hasluck, Cyzicus, Cambridge, 1910, p. 210-213 et L. ROBERT, in BCH, 102 (1978), p. 470-474.

36 Ci-dessus et note 31.

37 R. STIGLITZ, op. cit. (n. 1), p. 50-54.

38 PAUS., VIII, $31,8$.

39 Cf. M. JosT, op. cit. (n. 1), p. 346-349 et 333-334.

40 M. JosT, $R E A, 72$ (1970), p. 143. 
Les nuances précises attachées aux appellations de Mégalai Théai et de Sôteira nous échappent donc, et l'affirmation de Pausanias selon laquelle les Mégalai Théai, Déméter et Koré, sont honorées dans des rites où il faut voir "la réplique de ceux d'Éleusis» 41 vaut d'être prise en compte : après avoir rejeté l'hypothèse d'un couple syncrétique, venonsen à celle de «l'emprunt» à Éleusis.

Certes, l'affirmation de Pausanias ne doit pas être acceptée sans examen, car le Périégète, comme avant lui Hérodote, a tendance à rattacher ce qu'il décrit à des faits mieux connus de lui : ainsi à propos des deux canéphores placées devant les Mégalai Théai dans leur temple, Pausanias propose, contre l'avis général des gens de Mégalopolis, l'idée qu'il s'agirait d'Athéna et d'Artémis, compagnes de jeu de Koré lors de son enlèvement par Hadès ${ }^{42}$; cette version, qui n'est sans doute pas ancienne, témoigne de son goût pour les thèmes éleusiniens. C'est en revanche la tradition locale qu'il rapporte lorsqu'il écrit : «Il y a aussi, érigées dans un bâtiment, des effigies humaines, celle de Kallignotos et Mentas, Sosigénès et Pôlos, qui, d'après la tradition, implantèrent à Mégalopolis les mystères des Grandes Déesses, dont les rites sont la réplique $\left(\mu\left\llcorner\mu r^{\prime} \mu a \tau \alpha\right)\right.$ de ceux d'Éleusis" 43. Selon la tradition de Mégalopolis la fondation des mystères des Déesses était donc un fait historique, postérieur à la fondation de la cité, et commémoré par des statues honorifiques. Alors qu'à Phénéos par exemple la fondation d'un culte éleusinien est attribuée à un fondateur mythique ${ }^{44}$, ici nous sommes au IVe siècle, après 370/69.

Quand vécurent au juste ces fondateurs? On ne dispose pour le savoir d'aucun repère incontestable : une inscription du IIe s. av. J.C. ${ }^{45}$ avec le nom de Sosigénès fournit un argument pour l'historicité des personnages, mais elle ne les date pas, car le nom peut s'être perpétué dans la famille depuis le IVe siècle; quant à la date du IIIe-IIe s. av. J.C. que propose M. P. Nilsson ${ }^{46}$, il ne la justifie pas vraiment. Quelques témoignages épars semblent indiquer qu'Éleusis n'était pas inconnue dans le Péloponnèse déjà au Ve siècle, ce qui donne un terminus post

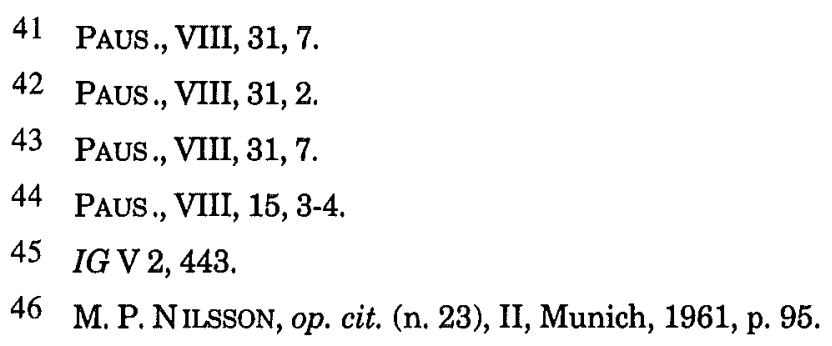


$q^{4} m^{47}$. On pourrait supposer que c'est dès la fin du IVe ou au début IIIe, au moment où était sans doute reconstruit le sanctuaire de Lykosoura ${ }^{48}$, que furent installées en pendant les Grandes Déesses; le sanctuaire de Mégalopolis et le réaménagement de celui de Lykosoura seraient contemporains. On observe d'ailleurs que sur plusieurs territoires arcadiens un culte de tonalité éleusinienne voisine avec un culte au particularisme local accusé : ainsi à Thelpousa et Phénéos; tel serait aussi le cas en Mégalopolitide où le culte éleusinien des Grandes Déesses contrasterait avec les tendances orgiastiques du culte du Mégaron de Lykosoura 49.

Quoi qu'il en soit au juste de la date de l'installation, ses modalités doivent être maintenant considérées. La mission que M.P. Nilsson attribue aux fondateurs se serait limitée à un remaniement des mystères $^{50}$ : cette interprétation n'est pas compatible avec le texte de

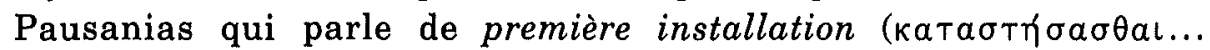

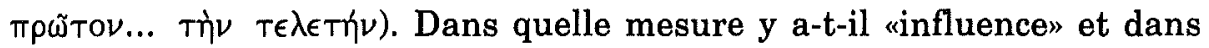
quelle mesure "emprunt"? En plusieurs sanctuaires d'Arcadie, il y a seulement une influence superficielle d'Éleusis : elle affecte l'épiclèse de Déméter (Éleusinia) à Basilis ou l'iconograpie du groupe cultuel à Lykosoura et à Thelpousa, mais à Lykosoura par exemple la célébration des mystères reste originale, avec ses mascarades animales, et ne prétend nullement se mouler sur les cérémonies éleusiniennes ${ }^{51}$; ainsi les canéphores, dont on a la reproduction dans les nombreuses statuettes en terre cuite offertes par les fidèles, portent un masque d'animal

47 Cf. M. JosT, op. cit. (n. 1), p. 354.

48 La date du temple et du groupe cultuel de Damophon est extrêmement discutée; mais la tendance actuelle est plutôt de la remonter : cf. M. JosT, op. cit. (n. 1), p. 174-176 et, récemment, P. THÉMÉLIS, Damophon von Messene -sein Werk im Lichte der neuen Ausgrabungen in $A K, 36$ (1993) 1, p. 24-40 et $O$

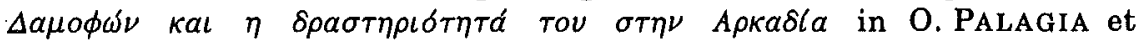
W. Coulson (eds), Sculpture from Arcadia and Laconia, Oxford, 1993, p. 99109; à la lumière d'une inscription trouvée à Messène, l'auteur date l'acmé de la carrière de Damophon entre 223 et 190 av. J.-C.

49 Thelpousa : PAus., VIII, 25, 2 et VIII, 25, 4-7; Phénéos : PAUS., VIII, 15, 1 et VIII, 15, 1-3; Lykosoura : PAUS., VIII, 37, 1-2.

50 M.P. NiLsson, Cults, Oracles and Politics in Ancient Greece, Lund, 1951, p. 20.

51 Cf. M. JosT, op. cit. (n. 1), p. 338-339 (Basilis); p. 327 et 301-302 (Lykosoura et Thelpousa); p. 331-333 (mystères de Lykosoura). 
rappelant les danses masquées représentées sur le voile de Despoina ${ }^{52}$. À Mégalopolis en revanche ce sont, comme à Éleusis, des jeunes filles qui, placées «devant le groupe central des Grandes Déesses», commémorent la canéphorie des "filles de Damophon» - quel que soit ici le personnage-53. D'ailleurs Pausanias, qui note les particularités iconographiques du groupe cultuel de Lykosoura, ne nomme pas les rites éleusiniens à propos des mystères des Déesses.

Sur le contenu des mystères à Mégalopolis, Pausanias est comme de coutume parfaitement discret; mais on observera que la formule $T \grave{d}$

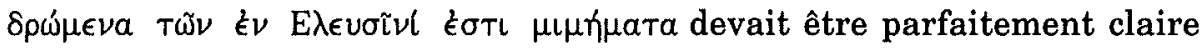
pour ses lecteurs initiés et n'appelait pas davantage de commentaire. Il s'agissait d'une "réplique» éleusinienne et il n'est pas jusqu'au nom des fonctions sacerdotales qui n'ait été calqué sur Éleusis : un décret pris par les Achéens à Lykosoura cite un certain Saon, «hiérophante des Grandes Déesses", "qui descend des hiérophantes fondateurs des mystères» 54 ; le hiérophante était on le sait un desservant essentiel à Éleusis. Tout porte donc à croire que les Mégalai Théai recevaient le même cérémonial que les Théai éleusiniennes et n'étaient donc guère différentes : il y a bien «emprunt».

Quel est le rôle d'Éleusis dans un pareil phénomène ? Il est peu vraisemblable que les grandes familles éleusiniennes aient souhaité établir des filiales de leur sanctuaire. Celui-ci, comme l'a récemment souligné $W$. Burkert ${ }^{55}$, comportait un certain nombre de caractères directement liés au site et impossibles à concevoir ailleurs (puits Kallirhoé, sanctuaire de Plouton en relation avec la geste de Déméter et Koré). Il faut plutôt voir dans le sanctuaire des Grandes Déesses, comme dans les nombreux sanctuaires de Déméter Eleusinia, un emprunt par la cité de Mégalopolis à un culte particulèrement renommé, fait pour rehausser son propre prestige religieux.

Ainsi le sanctuaire des Grandes Déesses, s'il offre plusieurs exemples "d'emprunts", tant à la chôra qu'à Éleusis, ne présente qu'un

\footnotetext{
52 Ibid, p. 332-333.

53 PAUS., VIII, 31, 2.

54 IG V 2, 517.

55 W. BuRKerT, Les cultes à mystères dans l'antiquité, Paris, 1992, p. 45. Cf. déjà R. Osborne, Demos : the Discovery of Classical Attika, Cambridge,1985, p. 154-177, pour Brauron et Éleusis.
} 
culte syncrétique au sens propre, celui de Zeus Philios. Pour le reste, on note une juxtaposition de cultes très différents les uns des autres : il ne s'agit pas de ce «mélange» qui modifie la substance des modèles dans les vrais syncrétismes et il paraît difficile de suivre $R$. Bastide ${ }^{56}$ qui parle de syncrétisme "écologique» - disons topographique - lorsque des images divines sont juxtaposées dans un même sanctuaire : l'acception trop générale donnée au terme de "syncrétisme» masque ce qu'il peut y avoir d'aléatoire dans la présence d'une divinité à côté d'une autre. C'est donc le terme d'«emprunt» qui paraît le mieux convenir pour définir les modalités de la mise en place de nouveaux cultes à Mégalopolis, et il n'y a rien là que de normal pour une cité qui cherche à se construire sur le modèle traditionnel, en s'appuyant autant que possible sur le passé religieux de la région et sur des modèles comme Éleusis dont la valeur fédératrice était déjà largement prouvée.

Université de Lille III

Madeleine JosT

F - 59653 VILLENEUVE D'ASCQ Cedex

56 R. BASTIDE, Les religions a fro-brésiliennes, Paris, 1960, p. 380-381. 